\title{
A Potentiometric Evaluation of Stability Constants of Two-Step Overlapping Equilibria via a Bilogarithmic Hyperbolic Cosine Method
}

\author{
Samuel Beaumont, Julia Martin, Agustin G. Asuero* \\ Department of Analytical Chemistry, University of Seville, Seville, Spain \\ Email: *asuero@us.es
}

Received 7 February 2016; accepted 20 June 2016; published 23 June 2016

Copyright (C) 2016 by authors and Scientific Research Publishing Inc.

This work is licensed under the Creative Commons Attribution International License (CC BY).

http://creativecommons.org/licenses/by/4.0/

(c) (i) Open Access

\begin{abstract}
A bilogarithmic hyperbolic cosine method for the evaluation of overlapping formation constants at varying (or fixed) ionic strength is devised in this paper and applied to data reported in the analytical literature, i.e. succinic acid system, Cu(II)-glycine system and Ag(I)-aminobutan-1-ol system. The method is based on the linearization of the formation function $\tilde{n}=f(p H)$ or $\tilde{n}=f(p L)$ data. A theoretical slope of unity should be obtained thus proving the correctness of the assumed equilibria. An additional advantage of the bilogarithmic method proposed is that it provides a closed scale representation of $\mathrm{Y}$ and $\mathrm{X}$ unlike other plots. This paper forms part of an investigation into the uses of bilogarithmic methods and hyperbolic functions in parameter estimation. Methods based on the application of spectrophotometric measurements have been the subject of recent studies.
\end{abstract}

\section{Keywords}

Formation Acidity Constants, Overlapping Equilibria, Potentiometric Measurements, Bilogarithmic Hyperbolic Cosine Method

\section{Introduction}

The exact determination of the thermodynamic formation constants of many dibasic acids is complicated by the overlapping [1]-[4] of the successive ionization steps. A great many methods have been derived [5]-[7] for the potentiometric evaluation of formation constants of two-step simultaneous equilibria. Of them, methods based on the formation function [8]-[12], $\tilde{n}=\mathrm{f}(\mathrm{pH})$, have been, undoubtedly, the most widely applied. The present pa-

\footnotetext{
${ }^{*}$ Corresponding author.
}

How to cite this paper: Beaumont, S., Martin, J. and Asuero, A.G. (2016) A Potentiometric Evaluation of Stability Constants of Two-Step Overlapping Equilibria via a Bilogarithmic Hyperbolic Cosine Method. Journal of Analytical Sciences, Methods and Instrumentation, 6, 33-43. http://dx.doi.org/10.4236/jasmi.2016.62005 
per describes a procedure for the study of stepwise equilibria in potentiometric titration, which is also based on Bjerrum's function. Data $(\tilde{n}, p H)$ are linearized according to a bilogarithmic mathematical model via a hyperbolic cosine method relationship. The treatment of the $(\tilde{n}, p H)$ data by the procedure derived in this paper does not require that the ionic strength is maintained constant by addition of inert salt. This paper forms part of an investigation [9] [13] into the uses of bilogarithmic methods and hyperbolic functions in parameter estimation. Methods based on the application of spectrophotometric measurements have been the subject [14]-[16] of recent studies.

\section{Theory}

For a diprotic acid $\mathrm{H}_{2} \mathrm{R}$, the average proton number [7] [9] [10] [17] [18] (the average number of proton bound per R) is given by

$$
\tilde{n}=\frac{[H R]+2\left[H_{2} R\right]}{[R]+[H R]+\left[H_{2} R\right]}
$$

where charges have been omitted for convenience. The stepwise thermodynamic formation constants of the acid is defined by

$$
\begin{gathered}
K_{1}^{T}=\frac{(H R)}{(H)(R)}=\frac{[H R] f_{1}}{(H)[R] f_{0}} \\
K_{2}^{T}=\frac{\left(H_{2} R\right)}{(H)(H R)}=\frac{\left[H_{2} R\right] f_{2}}{(H)[H R] f_{1}}
\end{gathered}
$$

where parenthesis indicate activities and braces concentrations; $f_{2}, f_{1}$ and $f_{0}$ being the activity coefficients of the species $\mathrm{H}_{2} \mathrm{R}, \mathrm{HR}$ and $\mathrm{R}$, respectively.

By combining Equations ((1)-(3)) we get

$$
\tilde{n}=\frac{K_{1}^{T} \frac{f_{0}}{f_{1}}(H)+2 K_{1}^{T} K_{2}^{T} \frac{f_{0}}{f_{2}}(H)^{2}}{1+K_{1}^{T} \frac{f_{0}}{f_{1}}(H)+K_{1}^{T} K_{2}^{T} \frac{f_{0}}{f_{2}}(H)^{2}} .
$$

On rearrangement Equation (4), we obtain

$$
\tilde{n}+(\tilde{n}-1) K_{1}^{T} \frac{f_{0}}{f_{1}}(H)+(\tilde{n}-2) K_{2}^{T} \frac{f_{0}}{f_{2}}(H)^{2}=0 .
$$

Two different situations will be considered in that follows depending whether the proton number values were lower or higher than the unity.

\subsection{Procedure for Average Number Values Lower Than the Unity}

By dividing Equation (5) by $(H)^{3 / 2}$, a further rearrangement leads to

$$
\frac{\tilde{n}}{(H)^{3 / 2}}=\frac{1-\tilde{n}}{(H)^{1 / 2}} K_{1}^{T} \frac{f_{0}}{f_{1}}+(2-\tilde{n}) K_{1}^{T} K_{2}^{T} \frac{f_{0}}{f_{2}} .
$$

By multiplying and dividing the right hand of Equation (6) by

$$
x=\frac{\sqrt{f_{1} f_{2}}}{f_{0}} \frac{1}{\sqrt{(1-\tilde{n})(2-\tilde{n})}} \frac{1}{K_{1}^{T} \sqrt{K_{2}^{T}}}
$$

we get

$$
\frac{\tilde{n}}{(H)^{3 / 2}}=K_{1}^{T} \sqrt{K_{2}^{T}} \frac{f_{0}}{\sqrt{f_{1} f_{2}}} \sqrt{(1-\tilde{n})(2-\tilde{n})}\left(\frac{1}{\sqrt{(H)}} \sqrt{\frac{1-\tilde{n}}{2-\tilde{n}}} \sqrt{\frac{f_{2}}{f_{1}}} \frac{1}{\sqrt{K_{2}^{T}}}+\sqrt{(H)} \sqrt{\frac{2-\tilde{n}}{1-\tilde{n}}} \sqrt{\frac{f_{1}}{f_{2}}} \sqrt{K_{2}^{T}}\right) .
$$

Making 


$$
\mathrm{e}^{y}=\frac{1}{\sqrt{(H)}} \sqrt{\frac{1-\tilde{n}}{2-\tilde{n}}} \sqrt{\frac{f_{2}}{f_{1}}} \frac{1}{\sqrt{K_{2}^{T}}}
$$

and taking into account that

$$
\cosh y=\frac{\mathrm{e}^{y}+\mathrm{e}^{-y}}{2} .
$$

Equation (8) may be converted into

$$
\frac{\tilde{n}}{\sqrt{(1-\tilde{n})(2-\tilde{n})}} \frac{1}{(H)^{3 / 2}} \frac{\sqrt{f_{1} f_{2}}}{f_{0}}=2 K_{1}^{T} \sqrt{K_{2}^{T}} \cosh \left(\frac{\ln 10}{2}\left(\log \left(\frac{1-\tilde{n}}{2-\tilde{n}}\right)+\log \frac{f_{2}}{f_{1}}+p H-\log K_{2}^{T}\right)\right)
$$

where $\mathrm{pH}=-\log (H)$. By taking logarithmic on both sides of Equation (11), on rearranging we finally get

$$
\begin{aligned}
& \log \cosh \left(\frac{\ln 10}{2}\left(\log \left(\frac{1-\tilde{n}}{2-\tilde{n}}\right)+\log \frac{f_{2}}{f_{1}}+p H-\log K_{2}^{T}\right)\right) \\
& =-\left(\log K_{1}^{T}+\frac{\log K_{2}^{T}}{2}\right)+\left(\log \left(\frac{\tilde{n}}{\sqrt{(1-\tilde{n})(2-\tilde{n})}}\right)+\log \frac{\sqrt{f_{1} f_{2}}}{f_{0}}+\frac{3}{2} p H-\log 2\right)
\end{aligned}
$$

Thus, a representation of the left term of Equation (12) against the term into brackets of the right hand should give a straight line $\left(Y=a_{0}+a_{1} X\right)$, obtained by linear regression [19]-[22], whose slope is the unity and the intercept with the $\mathrm{X}$-axis is equal to $-\left(\log K_{1}^{T}+\log K_{2}^{T} / 2\right)$, from which the $\log K_{1}^{T}$ may be estimated as

$$
\log K_{1}^{T}=-\frac{a_{0}}{a_{1}}-\frac{\log K_{2}^{T}}{2} .
$$

The application of Equations ((12) and (13)) requires, however, the previous knowledge of $\log K_{2}^{T}$. Different values of $\log K_{2}^{T}$ may be assumed and the entire procedure then applied. The best value of $\log K_{2}^{T}$ may be taken as that satisfies an optimization criterion, e.g. that minimizes the mean quadratic error (MQE) in $\tilde{n}$ measurements

$$
\sigma=\sqrt{\frac{\sum\left(\tilde{n}_{\text {exp }}-\tilde{n}_{\text {calc }}\right)^{2}}{N}}
$$

where $N$ is the number of data pairs, and $\tilde{n}$ is calculated from Equation (4) once both $\log K$ values are known, This task is easily carried out with the aid of an Excel spreadsheet.

In those cases in which the ionic strength is held constant by addition of an inert salt, e.g. potassium chloride or potassium nitrate $0.1 \mathrm{M}$, Equation (12) is converted into

$$
\begin{aligned}
& \log \left[\cosh \left(\frac{\ln 10}{2}\left(\log \left(\frac{1-\tilde{n}}{2-\tilde{n}}\right)+p H-\log K_{2}^{B}\right)\right)\right] \\
& =-\left(\log K_{1}^{B}+\frac{\log K_{2}^{B}}{2}\right)+\left(\log \left(\frac{\tilde{n}}{\sqrt{(1-\tilde{n})(2-\tilde{n})}}\right)+\frac{3}{2} p H-\log 2\right)
\end{aligned}
$$

where $K_{1}^{B}$ and $K_{2}^{B}$ are mixed or Bronsted constants, whose dependence on ionic strength can be expressed by

$$
\begin{gathered}
K_{1}^{B}=\frac{[H R]}{(H)[R]}=K_{1}^{T} \frac{f_{0}}{f_{1}}=\frac{K_{1}^{c}}{f_{H}} \\
K_{2}^{B}=\frac{\left[H_{2} R\right]}{(H)[H R]}=K_{2}^{T} \frac{f_{1}}{f_{2}}=\frac{K_{2}^{c}}{f_{H}},
\end{gathered}
$$

$K_{1}^{c}$ and $K_{2}^{c}$ are the stoicheiometric constants and $f_{\mathrm{H}}$ the activity factor of hydrogen ion. Note that the $\tilde{n}$ values 
when ionic strength is held constant are given by

$$
\tilde{n}=\frac{K_{1}^{B}+2 K_{1}^{B} K_{2}^{B}(H)^{2}}{1+K_{1}^{B}(H)+K_{1}^{B} K_{2}^{B}(H)^{2}} .
$$

\subsection{Procedure for Average Number Values Greater Than Unity}

In these situations, by dividing Equation (5) by $(H)^{1 / 2}$, on rearrangement we get

$$
(2-\tilde{n}) K_{1}^{T} K_{2}^{T} \frac{f_{0}}{f_{2}}(H)^{3 / 2}=(\tilde{n}-1) K_{1}^{T} \frac{f_{0}}{f_{1}} \sqrt{(H)}+\frac{\tilde{n}}{\sqrt{(H)}} .
$$

By multiplying through

$$
\frac{1}{\sqrt{\tilde{n}(\tilde{n}-1)}} \sqrt{\frac{f_{1}}{f_{0}}} \frac{1}{\sqrt{K_{1}^{T}}}
$$

Equation (19) is converted into

$$
\frac{2-\tilde{n}}{\sqrt{\tilde{n}(\tilde{n}-1)}} \frac{\sqrt{f_{0} f_{1}}}{f_{2}} \sqrt{K_{1}^{T}} K_{2}^{T}(H)^{3 / 2}=\left(\sqrt{\frac{\tilde{n}-1}{\tilde{n}}} \sqrt{\frac{f_{0}}{f_{1}}} \sqrt{K_{1}^{T}} \sqrt{(H)}+\sqrt{\frac{\tilde{n}}{\tilde{n}-1}} \sqrt{\frac{f_{1}}{f_{0}}} \frac{1}{\sqrt{K_{1}^{T}}} \frac{1}{\sqrt{(H)}}\right) .
$$

Taking into account the definition of hyperbolic cosine first and taking decadic logarithms on both sides of the resulting equation then, a posterior rearrangement leads to

$$
\begin{aligned}
& \log \left[\cosh \left(\frac{\ln 10}{2}\left(\log \left(\frac{\tilde{n}-1}{\tilde{n}}\right)+\log \frac{f_{0}}{f_{1}}-p H+\log K_{1}^{T}\right)\right)\right] \\
& =\left(\log K_{2}^{T}+\frac{\log K_{1}^{T}}{2}\right)+\left(\log \left(\frac{2-\tilde{n}}{\tilde{n}(\tilde{n}-1)}\right)+\log \frac{\sqrt{f_{0} f_{1}}}{f_{2}}-\frac{3}{2} p H-\log 2\right)^{.}
\end{aligned}
$$

When the left term of Equation (22) is plotted against the term into brackets of the right hand, a straight line $\left(Y=a_{0}+a_{1} X\right)$ of unity slope should be obtained, from which the value of $\log K_{2}^{T}$ may be estimated as

$$
\log K_{2}^{T}=\frac{a_{0}}{a_{1}}-\frac{\log K_{1}^{T}}{2} .
$$

Nevertheless, before Equation (22) can be applied, $\log K_{1}^{T}$ must be known. A procedure analogous to that suggested in the previous section may be followed in order to circumvent this difficulty.

If the ionic strength is maintained constant during the titration then

$$
\begin{aligned}
& \log \left[\cosh \left(\frac{\ln 10}{2}\left(\log \left(\frac{\tilde{n}-1}{\tilde{n}}\right)-p H+\log K_{1}^{B}\right)\right)\right] \\
& =\left(\log K_{2}^{B}+\frac{\log K_{1}^{B}}{2}\right)+\left(\log \left(\frac{2-\tilde{n}}{\sqrt{\tilde{n}(\tilde{n}-1)}}\right)-\frac{3}{2} p H-\log 2\right)
\end{aligned}
$$

The basis of this discussion has been protonation reactions, but the same principles apply for metal complexation reactions $\mathrm{M}+\mathrm{L}=\mathrm{ML}$ and $\mathrm{ML}+\mathrm{L}=\mathrm{ML}_{2}$

$$
\begin{gathered}
K_{1}=\frac{[M L]}{[M][L]} \\
K_{2}=\frac{\left[M L_{2}\right]}{[M L][L]}
\end{gathered}
$$

being the formation function or Bjerrum index in this case 


$$
\tilde{n}=\frac{[M L]+2\left[M L_{2}\right]}{[M]+[M L]+\left[M L_{2}\right]}=\frac{K_{1}[L]+2 K_{1} K_{2}[L]^{2}}{1+K_{1}[L]+K_{1} K_{2}[L]^{2}} .
$$

\subsection{Ionic Strength Expression}

Taking into account that $V_{0}$ millilitres of the diprotic acid $\mathrm{H}_{2} \mathrm{R}$ at a concentration $C_{A}$ moles/liter, haven been titrated with a volume $V$ of titrant, e.g. a strong monoacid base $\mathrm{BOH}$, of concentration $C_{B}$ moles/liter, the computation of ionic strength may be made assuming the Speakman [23] expression corrected by the volume, as a first approximation. Then, if $C_{B} V<C_{A} V_{0}$

$$
I=\frac{1}{2}([H]+[B]+[H R])=\frac{1}{2}\left([H]+2 \frac{C_{B} V}{V_{0}+V}\right) .
$$

In those cases in which $C_{B} V>C_{A} V_{0}$ we get

$$
I=\frac{1}{2}([H]+[B]+[H R]+2[R])=\frac{1}{2}\left([H]+2\left(\frac{2 C_{B} V-C_{A} V_{0}}{V_{0}+V}\right)\right) .
$$

The Debye-Hückel equation [24]-[26] (or other more sophisticated one) may be employed for the ionic activity coefficients and unity assumed for the activity of the uncharged molecule $\mathrm{H}_{2} \mathrm{R}$

$$
\log f_{i}=-\frac{A \sqrt{I}}{1+B \ddot{a} \sqrt{I}}
$$

where $A$ and $B$ are constants of the Debye-Hückel theory, and $\ddot{a}$ is the so-called ion-size parameter, or some extended form of the empirical Debye-Hückel equation as the Davies equation [27]. The activity coefficient may be evaluated if required by standard iteration to constant $f_{i}$.

\subsection{Error Analysis}

In those cases in which $\tilde{n}<1$, the straight line intersect the $\mathrm{X}$-axis at the point

$$
\log K_{1}^{T}+\frac{\log K_{2}^{T}}{2}=-\frac{a_{0}}{a_{1}}
$$

from which we may evaluate the value of $\log K_{1}^{T}$ once the value of $\log K_{2}^{T}$ is known.

By applying the law of random error propagation [28] we get

$$
s_{\log K_{1}^{T}}^{2}+\left(\frac{1}{2}\right)^{2} s_{\log K_{2}^{T}}^{2}=\frac{1}{a_{1}^{2}} s_{a_{0}}^{2}+\frac{a_{0}^{2}}{a_{1}^{4}} s_{a_{1}}^{2}-2 \frac{a_{0}}{a_{1}^{3}} \operatorname{cov}\left(a_{0}, a_{1}\right) .
$$

Taking into account [19]-[22] [28] the expressions for $\left(s_{a_{0}}\right)^{2},\left(s_{{\underline{a_{1}}}_{1}}\right)^{2}$ and $\operatorname{cov}\left(a_{0}, a_{1}\right)$ are given by

$$
s_{a_{1}}^{2}=\frac{s_{y / x}^{2}}{S_{X X}}, \quad s_{a_{0}}^{2}=\left(\frac{1}{N}+\frac{\bar{x}^{2}}{S_{X X}}\right) s_{y / x}^{2}, \quad \operatorname{cov}\left(a_{0}, a_{1}\right)=-\bar{x} \frac{s_{y / x}^{2}}{S_{X X}}, \quad s_{y / x}^{2}=\frac{S_{Y Y}-a_{1}^{2} S_{X X}}{N-2}
$$

where

$$
\bar{x}=\frac{\sum x}{N}, \quad S_{X X}=\sum x^{2}-\frac{\left(\sum x\right)^{2}}{N}, \quad S_{Y Y}=\sum y^{2}-\frac{\left(\sum y\right)^{2}}{N} .
$$

An estimate of the uncertainty of these calculations is given by

$$
s_{\log K_{1}^{T}}^{2}+\left(\frac{1}{2}\right)^{2} s_{\log K_{2}^{T}}^{2}=\left[\frac{1}{a_{1}^{2}}\left(\frac{1}{N}+\frac{\bar{x}^{2}}{S_{X X}}\right)+\frac{a_{0}^{2}}{a_{1}^{4} S_{X X}}+2 \frac{a_{0}}{a_{1}^{3}} \frac{\bar{x}}{S_{X X}}\right] s_{y / X}^{2}
$$

In those cases in which $\tilde{n}>1$ then

$$
\log K_{2}^{T}+\log K_{1}^{T} / 2=a_{0} / a_{1}
$$


and the application of the random error propagation law gives in this case

$$
s_{\log K_{2}}^{2}+\left(\frac{1}{2}\right)^{2} s_{\log K_{1}}^{2}=\left[\frac{1}{a_{1}^{2}}\left(\frac{1}{N}+\frac{\bar{x}^{2}}{S_{X X}}\right)+\frac{a_{0}^{2}}{a_{1}^{4} S_{X X}}+2 \frac{a_{0}}{a_{1}^{3}} \frac{\bar{x}}{S_{X X}}\right] s_{y / x}^{2} .
$$

\subsection{Choice of Starting Values}

Two principal difficulties should be self-evident. Primarily the present analysis requires a prior estimate of the individual stability constants. On this respect, preliminary values of $\log K_{1}^{T}$ and $\log K_{2}^{T}$ may be evaluated [9] [29] from Equations ((35) and (36)) by considering three well defined points on the titration curve at $\tilde{n}=0.5,1.0$, and 1.5

$$
\begin{gathered}
\log K_{1}^{T}=p H_{1.0}+2 \sqrt{3} \sinh (\ln (\Delta p H)-\log \sqrt{3}) \\
\log K_{2}^{T}=p H_{1.0}-2 \sqrt{3} \sinh (\ln (\Delta p H)-\log \sqrt{3})
\end{gathered}
$$

where

$$
\Delta p H=p H_{\tilde{n}=0.5}-p H_{\tilde{n}=1.5}=2\left(p H_{\tilde{n}=0.5}-p H_{\tilde{n}=1.0}\right)=2\left(p H_{\tilde{n}=1.0}-p H_{\tilde{n}=1.5}\right) .
$$

Expressions (35) and (36) are only approximate because of the influence of varying ionic strength. In addition, it is always disadvantageous to calculate stability constant from a minimum amount of experimental data. As a matter of fact, however, even the $p H$ values of $\tilde{n}=0.5$ and $\tilde{n}=1.5$ may be taken as starting point for $\log K_{2}^{T}$ and $\log K_{1}^{T}$ values, respectively.

\section{Applications}

In order to check the usefulness of the method it has been applied to a variety of systems previously described in the literature. Systems chosen for study were representative of the most difficult experimental situation encountered in practice. All have log $\mathrm{K}$ values similar in magnitude thus being very suitable for the purpose of this work. Experimental details and $[p H, V]$ and $[p L, \tilde{n}]$ data employed are given in that follows:

I. Succinic acid [24]: $C_{\mathrm{R}}=0.005 \mathrm{M} ; V_{0}=100 \mathrm{~mL}, C_{\mathrm{B}}=0.1 \mathrm{M}(\mathrm{KOH}) ; \mathrm{T}=25^{\circ}$. Data $[V, p H]$ : [1.00, 3.677; 1.25, 3.767; 1.50, 3.853; 1.75, 3932; 2.00, 4.009; 2.25, 4.081; 2.50, 4.153; 2.75, 4.223; 3.00, 4.291; 3.25, 4.361; 3.75, 4.498, 4.00, 4.569; 6.00, 5.135; 6.25, 5.204; 6.50, 5.273; 6.75, 5.342; 7.00, 5.412; 7.25, 5.480; 7.50, 5.554; 7.75, 5.629; 8.00, 5.208; 8.25, 5.789; 8.50; 5.881; 8.75, 5.981; 9.00, 6.099].

II. Cu(II)-Glicine system [18] at T $=25^{\circ} \mathrm{C}$. Data [pL, ñ]: [8.667, 0.250; 8.607, 0.270; 8.549, 0.296; 8.492, 0.326; 8.423, 0.351; 8.358, 0.385; 8.294, 0.426; 8.221, 0.463; 8.150, 0.511; 8.076, 0.564; 7.993, 0.620; 7.902, $0.681 ; 7.803,0.749 ; 7.715,0.807 ; 7.630,0.872 ; 7.215,1.169 ; 7.084,1.251 ; 6.975,1.139 ; 6.838,1.425 ; 6.708$, $1.515 ; 6.565,1.606 ; 6.380,1.697 ; 6.192,1.788 ; 5.886,1.880]$.

III. Silver(I)-4-aminobutan-1-ol [30] [31] at T $=20^{\circ} \mathrm{C}$ and I $=0.5$. Data [pL, $\left.\tilde{n}\right]$ : [4.198, 0.261; 4.121, 0.327; 4.058, 0.392; 4.000, 0.458; 3.950, 0.523; 3.906, 0.589; 3.861, 0.654; 3.818, 0.719; 3.780, 0.785; 3.740, 0.850; 3.700, 0.915; 3.59, 1.110; 3.549, 1.110; 3.516, 1.238; 3.477, 1.303; 3.389, 1.429; 3.292, 1.553; 3.173, 1.671; $3.023,1.779 ; 2.824,1.862]$.

Figure 1 shows the application of the bilogarithmic hyperbolic cosine method (BHCM) to the succinic acid system. The residuals obtained were $[-+++-+---+-++]$ and $[++-+---+-+++-]$ for $\tilde{n}<1$ and $\tilde{n}>$ 1 , respectively, then show no special pattern. A well defined unity slope, $1.0009 \pm 0.0043$ and $0.9985 \pm 0.0024$, respectively, was obtained in both cases. The results obtained by means of the BHMC method are in good agreement with the values obtained by Albert and Serjeant [25] by applying a computerized FORTRAN method.

The ideal methodology devised for $\mathrm{H}_{2} \mathrm{R} / \mathrm{HR} / \mathrm{R}$ systems may be applied to simultaneous complex systems $\mathrm{ML}_{2} / \mathrm{ML} / \mathrm{M}$. In this case the data available are $(p L, \tilde{n})$. Figure 2 and Figure 3 show the application of the BHMC method to the $\mathrm{Cu}(\mathrm{II})$-glycine and $\mathrm{Ag}(\mathrm{I})-4$-aminobutan-1-ol systems, respectively. Irving and Rossotti (18) obtained for the $\mathrm{Cu}(\mathrm{II})$-glycine system (Table 1 ) values of $\log K_{1}$ of 8.12 to 8.16 and $\log K_{2}$ of 6.73 to 6.78 . The results obtained in this paper are [8.177 - 8.143] for $\log K_{1}$, and [6.772 to 6.645] for $\log K_{2}$. The values obtained for $\tilde{n}<1$ and $\tilde{n}>1$ differ in 0.034 and $0.127 \log$ units, for $\log K_{1}$ and $\log K_{2}$, respectively. The slopes of our method in both cases are close to $1(0.9787 \pm 0.0271$ for $\tilde{n}<1$ and $0.9878 \pm 0.0134$ for $\tilde{n}>1)$. 


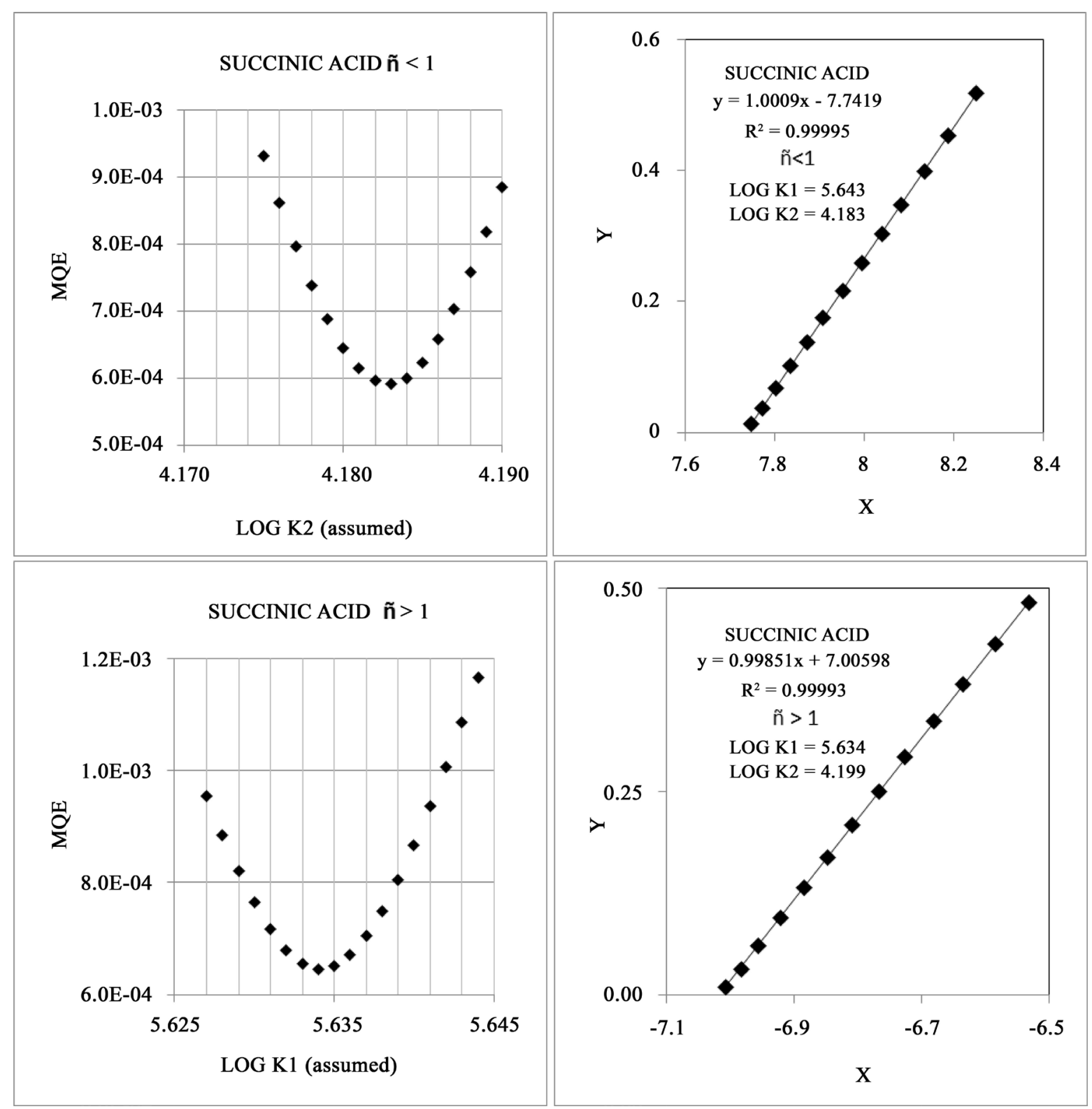

Figure 1. Top left: Mean quadratic error (MQE) as a function of $\log K_{2}$ assumed $(\tilde{n}<1)$. Top right: Bilogarithmic plot $(\tilde{n}<1)$ for the succinic acid system. Bottom left: Mean quadratic error (MQE) as a function of $\log K_{1}$ assumed $(\tilde{n}>1)$. Bottom right: Bilogarithmic plot $(\tilde{n}>1)$ for the succinic acid system.

A well defined slope $(1.0073 \pm 0.0136)$ was obtained for the system $A g(I)-4$-aminobutan-1-ol $(\tilde{n}>1)$ and values of $\log K_{1}$ and $\log K_{2}$ of $3.416 \pm 0.002$ and 3.896 (assumed), respectively. Lansbury et al. [30] and Unwin et al. [31] obtained values of 3.41 and 3.89, respectively, using computerized methods based on the use of weighted least squares, and response surfaces, respectively. The results obtained by applying the BHMC method proposed in this paper coincide with those provided by these authors. The Ag(I)-4-aminobutan-ol system, however, departs from a behaviour model at $\tilde{n}>1$ values.

\section{Conclusion}

A major goal of scientific experimentation is the discovery of relationships [32] among variables. The evaluation of stability constant by linearized plots on this respect seems to be more prevalent, probably owing to the transparency [33] of the methods used. Note that non-linear least squares are not always problem-free. Occasio- 
nally, problems arise [34] because of the choice of the data, initial estimates, convergence or multiple local minima, and all-typical of non-linear regression. A main advantage of the bilogarithmic method devised in this paper is that a theoretical slope of unity should be obtained this proving directly the correctness of the assumed equilibria. Significant deviation from this behaviour is indicative of more complicated phenomena. It is interesting to note
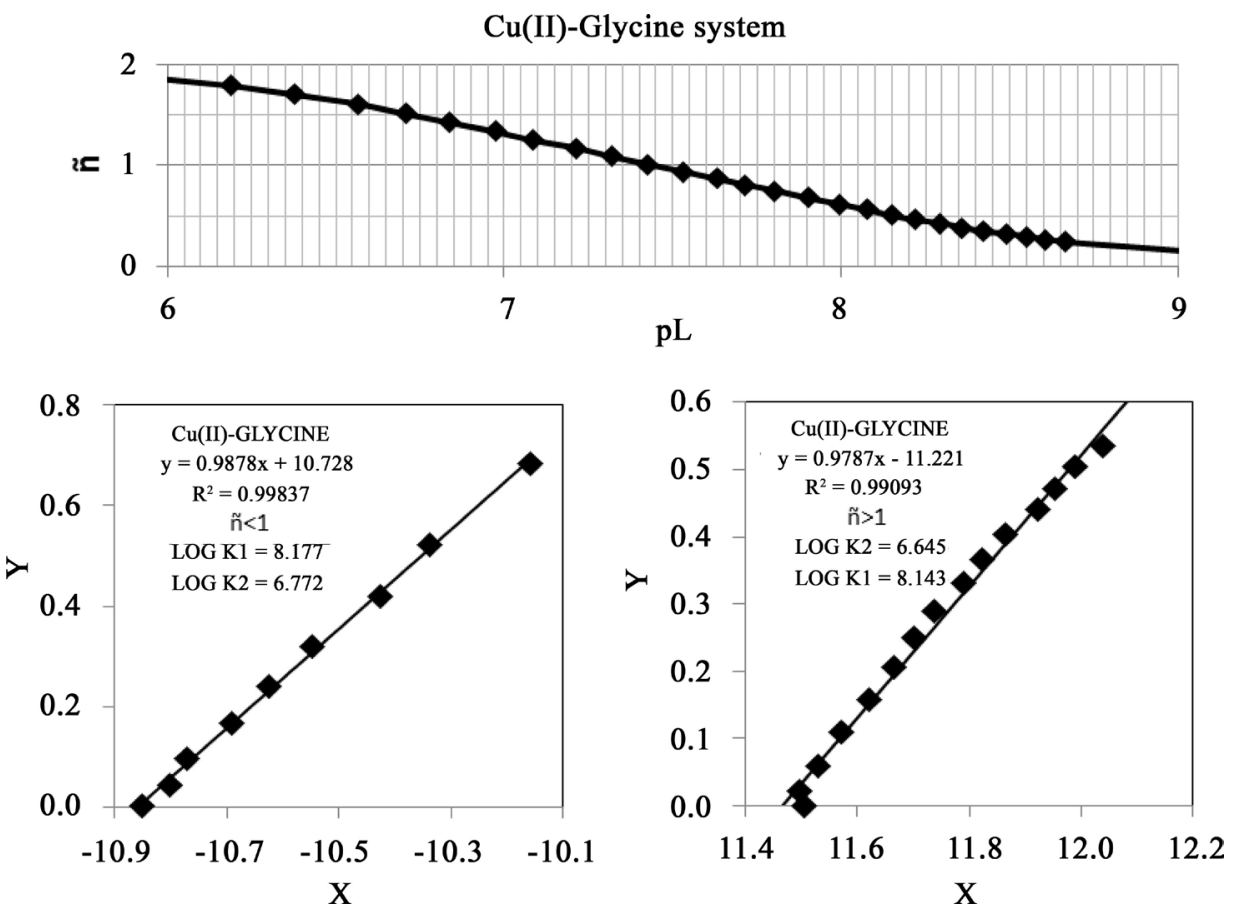

Figure 2. Top: Graphical representation for the ñ versus $\mathrm{pL}$ data. The curve in the figure is calculated with $\log K_{1}$ and $\log K_{2}$ given in Table 1 (bilogarithmic method). Bottom left and right: logarithmic plots.
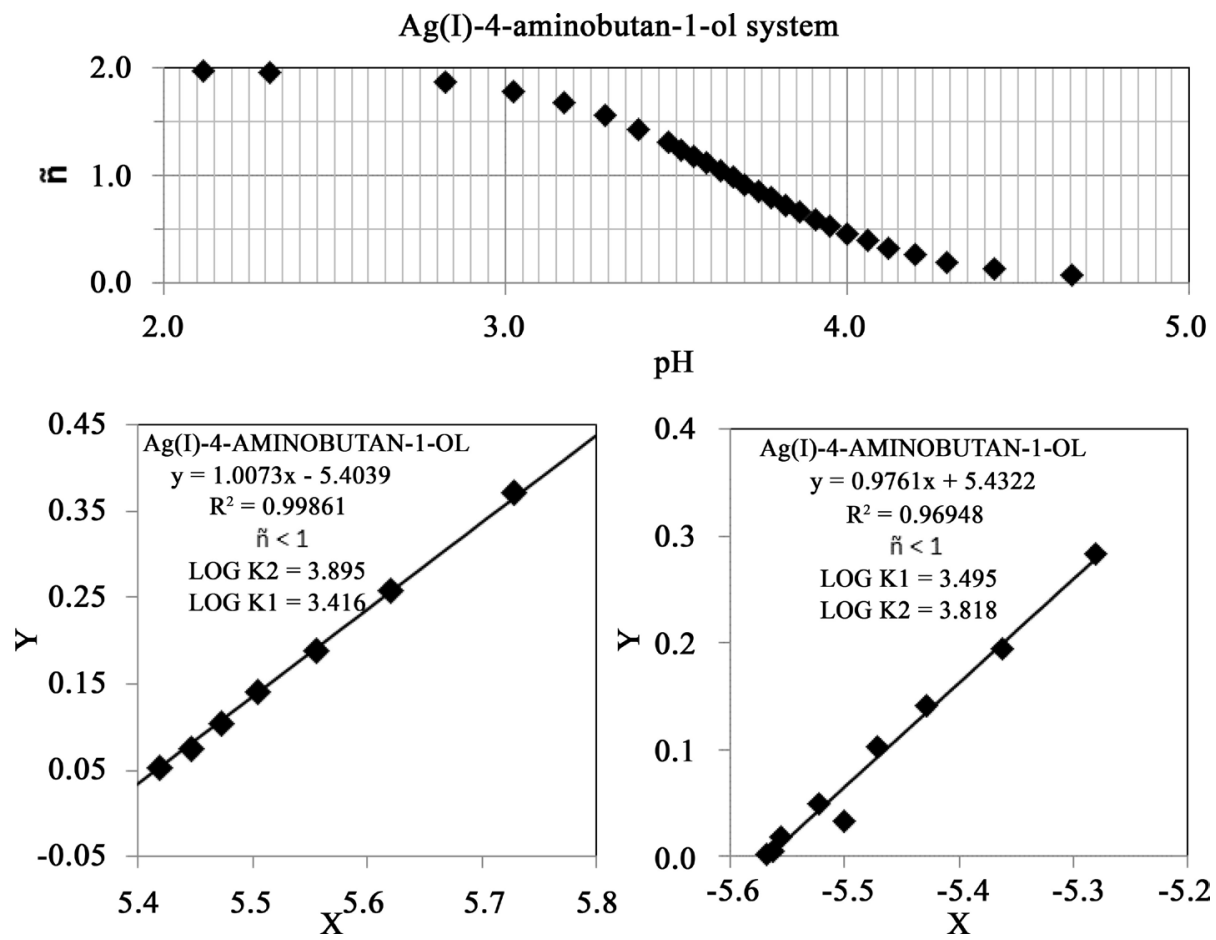

Figure 3. Top: Graphical representation for the $\tilde{n}$ versus pL data. Bottom left and right: Bilogarithmic plots. 
Table 1. Comparison of results obtained by different methods in the evaluation of formation constants.

\begin{tabular}{|c|c|c|c|c|}
\hline System & Method & $\log K_{1}$ & $\log K_{2}$ & Ref. \\
\hline \multirow{3}{*}{ Succinic acid } & Computer FORTRAN method & 5.634 & 4.200 & [24] \\
\hline & $\operatorname{BHCM}(\tilde{n}<1)$ & $5.643 \pm 0.008$ & 4.183 & This paper \\
\hline & $\operatorname{BHCM}(\tilde{n}>1)$ & 5.634 & $4.199 \pm 0.002$ & This paper \\
\hline \multirow{5}{*}{$\mathrm{Cu}(\mathrm{II})$-Glycine } & Successive approximation method & 8.16 & 6.73 & [18] \\
\hline & Correction term method & 8.13 & 6.78 & [18] \\
\hline & Least squares treatment & 8,12 & 6.77 & [18] \\
\hline & $\operatorname{BHCM}(\tilde{n}<1)$ & $8.143 \pm 0.009$ & 6.772 & This paper \\
\hline & $\operatorname{BCHM}(\tilde{n}>1)$ & 8.177 & $6.672 \pm 0.005$ & This paper \\
\hline \multirow{4}{*}{ Ag(I)-4-aminobutan-1-ol } & Weighted least squares method & 3.41 & 3.89 & [30] \\
\hline & Computer FORTRAN technique & 3.41 & 3.89 & [31] \\
\hline & $\operatorname{BCHM}(\tilde{n}<1)$ & $3.416 \pm 0.002$ & 3.896 & This paper \\
\hline & $\operatorname{BCHM}(\tilde{n}>1)$ & 3.495 & $3.818 \pm 0.007$ & This paper \\
\hline
\end{tabular}

that by applying other least-squares procedures, it is not possible to determine whether a given pH against fraction titrated curve is characterized only by the assumed reactions. In this respect, when the independent and dependent variables are varied over a number of orders of magnitudes, the points tend usually [17] to be bunched together. However, an additional advantage of the bilogarithmic method reported here provides a closed scale representation of $\mathrm{y}$ and $\mathrm{x}$, unlike other plots. The bilogarithmic hyperbolic tool devised here, for all reasons indicated above, constitutes an appropriate and useful mathematical model for the potentiometric study of simultaneous equilibria.

\section{References}

[1] Asuero, A.G. and Michałowski, T. (2011) Comprehensive Formulation of Titration Curves Referred to Complex Acid-Base Systems and Its Analytical Implications. Critical Reviews in Analytical Chemistry, 41, 151-187. http://dx.doi.org/10.1080/10408347.2011.559440

[2] Avdeef, A. (2012) pKa Determination. In: Absorption and Drug Development: Solubility, Permeability and Charge State, Chap. 3, 2nd Edition, Wiley, New York, Hoboken, NJ, 31-173.

[3] Asuero, A.G., Jimenez, J.L. and Navas, M.J. (1986) Mathematical Treatment of Absorbance versus pH Graphs of Polybasic Acids. Talanta, 33, 929-934. http://dx.doi.org/10.1016/0039-9140(86)80228-4

[4] Asuero, A.G., Jimenez, J.L. and Navas, M.J. (1986) Spectrophotometric Methods for the Evaluation of Acidity Constants: Numerical Methods for Two Step Overlapping Equilibria. Talanta, 33, 531-535. http://dx.doi.org/10.1016/0039-9140(86)80265-X

[5] Gonçalves, E.M. and Conceição, A.C.L. (2011) Constantes de acidez de ácidos dipróticos a partir de titulações potentiométricas: ilustração dos princípios do cálculo através da construção de um algoritmo muito simples. Quimica Nova, 34, 1074-1078. http://dx.doi.org/10.1590/S0100-40422011000600027

[6] Jano, I., Hardcastle, J., Jano, L., Bates, K.R. and McCreary, H.E. (2001) General Equation for Determining the Dissociation Constants of Polyprotic Acids and Bases from Additive Property. Part IV. Application to Potentiometric Data. Analytica Chimica Acta, 428, 309-321. http://dx.doi.org/10.1016/S0003-2670(00)01242-3

[7] Ramos, G.R. and Alvarez-Coque, M.C. (1989) Examination of the Least Squares Method Applied to the Evaluation of Physicochemical Parameters with Linearized Equations. Analytica Chimica Acta, 220, 145-153. http://dx.doi.org/10.1016/S0003-2670(00)80258-5

[8] Hurek, J. and Nackiewicz, J. (2013) A Simple Method for the Consecutive Determination of Protonation Constants through Evaluation of Formation Curves. Journal of Chemical Education, 90, 604-608. http://dx.doi.org/10.1021/ed300172a

[9] Asuero, A.G. (2007) Buffer Capacity of a Polyprotic Acid: First Derivative of the Buffer Capacity and pKa Values of Simple and Overlapping Equilibria. Critical Reviews in Analytical Chemistry, 37, 269-301. http://dx.doi.org/10.1080/10408340701266238

[10] Dimitrov, S.S. and Kamenski, D.I. (1997) Overall Stability Constants: Estimation by Non-Linear Least Squares Me- 
thods. Computers in Chemistry, 21, 167-173. http://dx.doi.org/10.1016/S0097-8485(96)00025-3

[11] Kraft, A. (2003) The Determination of the pKa of Multiprotic Weak Acids by Analyzing Potentiometric Acid-Base Titration Data with Difference Plots. Journal of Chemical Education, 80, 554-559. http://dx.doi.org/10.1021/ed080p554

[12] Moya-Hernandez, R., Rueda-Jackson, J.C., Ramirez, M.T., Vazquez, G.A., Havel, J. and Rojas-Hernández, A. (2002) Statistical Study of Distribution Diagrams for Two-Component Systems: Relationships of Means and Variances of the Discrete Variable Distributions with Average Ligand Number and Intrinsic Buffer Capacity. Journal of Chemical Education, 79, 389-392. http://dx.doi.org/10.1021/ed079p389

[13] Asuero, A.G. (1989) A Bilogarithmic Method for the Evaluation of Acidity Constants of Amphoteric Substances from Solubility Measurements. International Journal of Pharmaceutics, 52, 129-137. http://dx.doi.org/10.1016/0378-5173(89)90287-1

[14] Asuero, A.G. (2009) A Hyperbolic Sine Procedure for the Spectrophotometric Evaluation of Acidity Constants of Two-Step Overlapping Equilibria. Journal of Analytical Chemistry, 64, 1026-1030. http://dx.doi.org/10.1134/S1061934809100098

[15] Sayago, A. and Asuero, A.G. (2006) Spectrophotometric Evaluation of Stability Constants of 1:1 Weak Complexes from Continuous Variation Data. International Journal of Pharmaceutics, 321, 94-100. http://dx.doi.org/10.1016/j.ijpharm.2006.05.028

[16] Boccio, M., Sayago, A. and Asuero, A.G. (2006) A Bilogarithmic Method for the Spectrophotometric Evaluation of Stability Constants of 1:1 Weak Complexes from Mole Ratio Data. International Journal of Pharmaceutics, 318, $70-77$. http://dx.doi.org/10.1016/j.ijpharm.2006.03.026

[17] Rossotti, H. (1978) The Study of Ionic Equilibria, an Introduction. Longman, London.

[18] Irving, H. and Rossotti, H.S. (1953) Methods for Computing Successive Stability Constants from Experimental Formation Curves. Journal of the Chemical Society, 3397-3405. http://dx.doi.org/10.1039/jr9530003397

[19] Sayago, A., Boccio, M. and Asuero, A.G. (2004) Fitting Straight Lines with Replicated Observations by Linear Regression: The Least Squares Postulates. Critical Reviews in Analytical Chemistry, 34, 39-50. http://dx.doi.org/10.1080/10408340490273744

[20] Asuero, A.G., Sayago, A. and González, A. (2006) The Correlation Coefficient: An Overview. Critical Reviews in Analytical Chemistry, 36, 41-59. http://dx.doi.org/10.1080/10408340500526766

[21] Asuero, A. and Gonzalez, A.G. (1989) Some Observations on Fitting a Straight Line to Data. Microchemical Journal, 40, 216-225. http://dx.doi.org/10.1016/0026-265X(89)90073-8

[22] Asuero, A.G. and Gonzalez, A.G. (2007) Fitting Straight Lines with Replicated Observations by Linear Regression. III. Weighting Data. Critical Reviews in Analytical Chemistry, 37, 143-172. http://dx.doi.org/10.1080/10408340701244615

[23] Speakman, J.C. (1940) The Determination of the Thermodynamic Dissociation Constants of Dibasic Acids. Journal of Chemical Society, 855-859. http://dx.doi.org/10.1039/jr9400000855

[24] Albert, A. and Serjeant, E.P. (1984) The Determination of Ionization Constants, a Laboratory Manual. 3rd Edition, Chapman \& Hall, New York. http://dx.doi.org/10.1007/978-94-009-5548-6

[25] Litchinsky, D., Purdie, N., Tompson, M.B. and White, W.D. (1969) A Rigorous Solution to the Problem of Interfering Dissociation Steps in the Titration of Polybasic Acids. Analytical Chemistry, 41, 1726-1730. http://dx.doi.org/10.1021/ac60282a007

[26] Briggs, T.N. and Stuehr, J.E. (1975) Simultaneous Potentiometric Determination of Precise Equivalence Points and pK Values of Two- and Three-pK Systems. Analytical Chemistry, 47, 916-1920. http://dx.doi.org/10.1021/ac60362a012

[27] Christian, G.D., Dasgupta, P.K. and Schug, K.A. (2015) Analytical Chemistry. 7th Edition, Wiley, New York, 211217.

[28] Asuero, A.G., Gonzalez, G., de Pablos, F. and Ariza, J.L.G. (1988) Determination of the Optimum Working Range in Spectrophotometric Procedures. Talanta, 35, 131-137. http://dx.doi.org/10.1016/0039-9140(88)80127-9

[29] Asuero, A.G. (1990) Observations on the Computation of Acidity Constants of Two Step Overlapping Equilibria from the Half Values of the Formation Function. Analytical Letters, 23, 747-753. http://dx.doi.org/10.1080/00032719008052478

[30] Lansbury, R.C., Price, V.E. and Smetld, A.G. (1965) The Application of Weighted Least Squares Method to the Computation of Stability Constants. Journal of Chemical Society, 1896-1900. http://dx.doi.org/10.1039/jr9650001896

[31] Unwin, E.A., Beimer, R.G. and Fernando, Q. (1967) The Use of a Digital Computer for the Calculations of Successive Complex Formation. Analytica Chimica Acta, 39, 95-102. http://dx.doi.org/10.1016/s0003-2670(01)80459-1

[32] Connors, K.A. (1987) Binding Constants, the Measurement of Molecular Complex Stability. Wiley, New York. 
[33] Fuchs, H. and Gessner, R. (2001) The Result of Equilibrium-Constant Calculations Strongly Depends on the Evaluation Method Used and on the Type of Experimental Errors. Biochemical Journal, 359, 411-418. http://dx.doi.org/10.1042/bj3590411

[34] Nievergelt, Y. (1994) Exact Equations for the Equilibrium Constants of Single Complexes in Terms of Spectrophotometric Data. Analyst, 119, 145-151. http://dx.doi.org/10.1039/an9941900145 